\title{
Analytic Expressions for Electromagnetic Field Envelopes in a 1D Photonic Crystal
}

\author{
A. RudZińsKi* \\ Institute of Microelectronics and Optoelectronics \\ Warsaw University of Technology \\ Koszykowa 75, 00-665 Warszawa, Poland
}

(Received November 7, 2006)

\begin{abstract}
The subject of this paper is the derivation of expressions describing envelopes of electromagnetic fields inside a $1 \mathrm{D}$ photonic crystal. The presented approach is based on an effective resonator model, which treats each layer of the photonic crystal as a resonant cavity. The model is analytic and allows one to derive parameters characterizing the photonic crystal and express electric and magnetic fields as their function. It is general enough to take into account defects of the crystal or even be used for an aperiodic structure. In the end of the paper, a few results are provided for the case of a source of radiation inside a photonic crystal along with a short discussion.
\end{abstract}

PACS numbers: 42.70.Qs

\section{Motivation}

It has been in the late 1980s when photonic crystals have been recognized as a distinct group of materials with unique optical properties [1,2]. Their properties are associated with an optical band structure resembling the band structure of semiconductors in electronics, and therefore photonic crystals are sometimes called "semiconductors of light". Because semiconductors have played a very important role in science and technology, becoming the fundamentals of contemporary electronics, this similarity is the cause of great interest and expectations regarding photonic crystals. Indeed, photonic crystals have been used to build many optical devices - one can mention optical waveguides [3, 4], filters [5], optical switches [6], LEDs with increased efficiency [7], distributed Bragg reflector (DBR) and distributed feedback (DFB) lasers [8].

It is important to understand the physics of photonic crystals to develop new devices and improve already existing ones. In this paper, there is presented an effective resonator model of a 1D photonic crystal, which allows one to exam-

*e-mail: a.rudzinski@elka.pw.edu.pl 
ine a wide range of properties of this kind of structures - here it is employed for derivation of expressions for electromagnetic field envelopes inside a photonic crystal. The model is simple, analytic, and versatile - in particular, it can be used for photonic crystals with defects or chirped photonic crystals. This fact allows us to expect that this model can seriously contribute to the understanding of photonic crystals, and its predictions or conclusions drawn from it could be later used in investigations of more complicated photonic band-gap structures.

\section{Reflection and transmission coefficients}

A structure of a 1D photonic crystal considered in this paper is presented in Fig. 1. It is built of a finite number of dielectric layers with refractive index $n_{1}$ and width $a$ or refractive index $n_{2}$ and width $b$. The layers extend towards the infinity in directions perpendicular to the symmetry axis. A pair of two adjacent layers forms an elementary cell of the crystal. It is assumed that on both sides of the photonic crystal there are regions of space filled with uniform dielectrics.

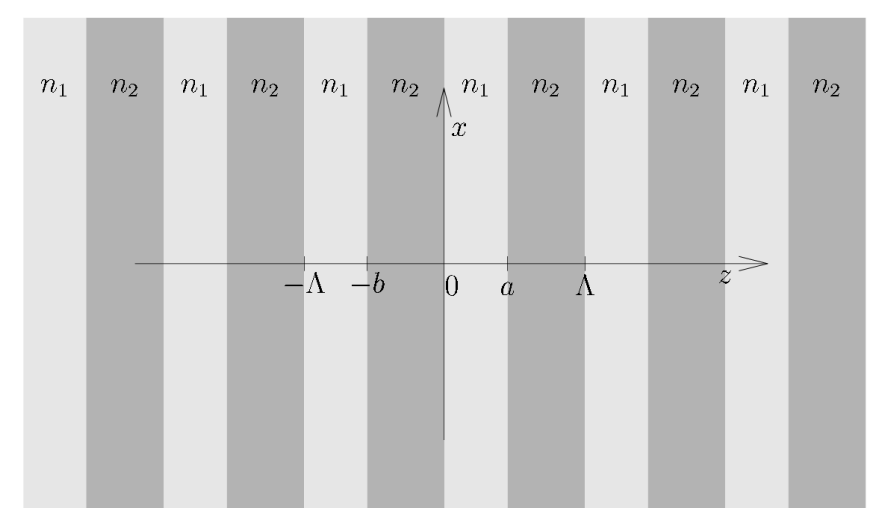

Fig. 1. A structure of a 1D photonic crystal.

The treatment of the photonic crystal in this paper is based on the effective resonator model, which employs the translation matrix method, introduced for infinite 1D photonic crystals in [9], and adapted for finite 1D photonic crystals in [10]. Let $\boldsymbol{n}$ be a versor parallel to the symmetry axis of the photonic crystal and $\boldsymbol{k}$ be the wave vector of a plane wave. These two vectors determine the plane of incidence, which makes it possible to distinct two plane-wave polarizations: TE, if the vector of electric field is perpendicular to the plane of incidence and TM, in which case the vector of magnetic field is perpendicular to the plane of incidence. Versors indicating the direction of electric field vector for these polarizations can be defined as the following:

$$
\boldsymbol{e}_{k \mathrm{TE}}=\frac{\boldsymbol{k} \times \boldsymbol{n}}{|\boldsymbol{k} \times \boldsymbol{n}|}, \quad \boldsymbol{e}_{k \mathrm{TM}}=\frac{\boldsymbol{e}_{k \mathrm{TE}} \times \boldsymbol{k}}{k}
$$

(this definition is not suitable for $\boldsymbol{k} \| \boldsymbol{n}$, in which case any versor perpendicular 
to $\boldsymbol{k}$ can be chosen for $\left.\boldsymbol{e}_{k \mathrm{TE}}\right)$. In every photonic crystal layer, for each of the polarizations, the Maxwell equations have solutions in the form of

$$
U_{i}(\boldsymbol{r})=a_{i} \mathrm{e}^{\mathrm{i} \boldsymbol{k} \boldsymbol{r}}+b_{i} \mathrm{e}^{\mathrm{i} \boldsymbol{k}_{*} \boldsymbol{r}}
$$

where $i$ indexes layers (and the regions of space outside the crystal), $U_{i}$ denotes the amplitude of electric field for TE polarization or magnetic field for TM polarization (polarization index is omitted in this part of derivation), and $\boldsymbol{k}_{*}$ is the wave vector of reflected plane wave, related to the incident plane-wave wave vector $\boldsymbol{k}$ with the equation

$$
\boldsymbol{k}_{*}=\boldsymbol{k}-2(\boldsymbol{k} \cdot \boldsymbol{n}) \boldsymbol{n} .
$$

It is assumed that each layer has a separate coordinate system associated with it, and each of these coordinate systems can be transferred to any other of them by only one translation along its $z$ axis (they have "common" $x$ and $y$ axes), and has its origin on the layer's boundary plane which has the lesser coordinate $z$ - an exemplary coordinate system is shown in Fig. 1. The same applies to coordinate systems associated with the regions outside the crystal, each of which has only one boundary plane, and the origin of the proper coordinate system is placed on the plane.

Amplitudes $a_{i}$ and $b_{i}$ obey the continuity conditions, which can be conveniently written in the matrix form

$$
\left(\begin{array}{c}
a_{i+1} \\
b_{i+1}
\end{array}\right)=m_{i+1, i}\left(\begin{array}{c}
a_{i} \\
b_{i}
\end{array}\right)
$$

where matrix $m_{i+1, i}$ depends on polarization. For TE

$$
m_{i+1, i}^{(\mathrm{TE})}=\frac{1}{2}\left(\begin{array}{ll}
\left(1+\frac{k_{z}^{(i)}}{k_{z}^{(i+1)}}\right) \mathrm{e}^{\mathrm{i} k_{z}^{(i)} L_{z}^{(i)}} & \left(1-\frac{k_{z}^{(i)}}{k_{z}^{(i+1)}}\right) \mathrm{e}^{-\mathrm{i} k_{z}^{(i)} L_{z}^{(i)}} \\
\left(1-\frac{k_{z}^{(i)}}{k_{z}^{(i+1)}}\right) \mathrm{e}^{\mathrm{i} k_{z}^{(i)} L_{z}^{(i)}} & \left(1+\frac{k_{z}^{(i)}}{k_{z}^{(i+1)}}\right) \mathrm{e}^{-\mathrm{i} k_{z}^{(i)} L_{z}^{(i)}}
\end{array}\right),
$$

while for TM

$$
m_{i+1, i}^{(\mathrm{TM})}=\frac{1}{2}\left(\begin{array}{ll}
\left(1+\frac{n_{(i+1)}^{2} k_{z}^{(i)}}{n_{(i)}^{2} k_{z}^{(i+1)}}\right) \mathrm{e}^{\mathrm{i} k_{z}^{(i)} L_{z}^{(i)}} & \left(1-\frac{n_{(i+1)}^{2} k_{z}^{(i)}}{n_{(i)}^{2} k_{z}^{(i+1)}}\right) \mathrm{e}^{-\mathrm{i} k_{z}^{(i)} L_{z}^{(i)}} \\
\left(1-\frac{n_{(i+1)}^{2} k_{z}^{(i)}}{n_{(i)}^{2} k_{z}^{(i+1)}}\right) \mathrm{e}^{\mathrm{i} k_{z}^{(i)} L_{z}^{(i)}} & \left(1+\frac{n_{(i+1)}^{2} k_{z}^{(i)}}{n_{(i)}^{2} k_{z}^{(i+1)}}\right) \mathrm{e}^{-\mathrm{i} k_{z}^{(i)} L_{z}^{(i)}}
\end{array}\right),
$$

where $k_{z}^{(i)}$ is the $z$ component of wave vector in the $i$-th layer, and $L_{z}^{(i)}$ is the layer's width or 0 if index $i$ corresponds to any of regions outside the crystal. The inverse relation is

$$
\left(\begin{array}{c}
a_{i-1} \\
b_{i-1}
\end{array}\right)=w_{i-1, i}\left(\begin{array}{c}
a_{i} \\
b_{i}
\end{array}\right),
$$

with matrix $w_{i-1, i}$ defined as

$$
\begin{aligned}
& w_{i-1, i}= \\
& \left(\begin{array}{cc}
\mathrm{e}^{-\mathrm{i} k_{z}^{(i-1)} L_{z}^{(i-1)}} & 0 \\
0 & \mathrm{e}^{\mathrm{i} k_{z}^{(i-1)} L_{z}^{(i-1)}}
\end{array}\right) m_{i-1, i}\left(\begin{array}{cc}
\mathrm{e}^{-\mathrm{i} k_{z}^{(i)} L_{z}^{(i)}} & 0 \\
0 & \mathrm{e}^{\mathrm{i} k_{z}^{(i)} L_{z}^{(i)}}
\end{array}\right) .
\end{aligned}
$$


It follows from (4a) that amplitudes $a_{N}$ and $b_{N}$ are related to $a_{0}$ and $b_{0}$ :

$$
\left(\begin{array}{l}
a_{N} \\
b_{N}
\end{array}\right)=m_{N, 0}\left(\begin{array}{l}
a_{0} \\
b_{0}
\end{array}\right)
$$

where matrix $m_{j, i}$ is the product of continuity conditions matrices for layers between the $i$-th and the $j$-th

$$
m_{j, i}=m_{j, j-1} \ldots m_{i+2, i+1} m_{i+1, i}, \quad j>i .
$$

If the index $N$ corresponds to the dielectric behind the structure, then $b_{N}=0$, because there is no reflection from the infinity. In this case it is possible to find $b_{0}$ as a function of $a_{0}$, which allows one to calculate the electric field reflection coefficient of the structure built of the layers with indices from 1 to $N-1$ and the dielectric behind them

$$
r_{N, 0}=\frac{b_{0} \mathrm{e}^{-\mathrm{i} k_{z}^{(0)} L_{z}^{(0)}}}{a_{0} \mathrm{e}^{\mathrm{i} k_{z}^{(0)} L_{z}^{(0)}}}=-\frac{\left(m_{N, 0}\right)_{21}}{\left(m_{N, 0}\right)_{22}} \mathrm{e}^{-2 \mathrm{i} k_{z}^{(0)} L_{z}^{(0)}}
$$

(there is no change of sign for TM polarization in the above expression, because it is already incorporated in the polarization versor (1)). Similarly, the electric field transmission coefficient of the structure can be found. The formula for this coefficient depends on polarization, for TE one obtains

$$
t_{N, 0}^{(\mathrm{TE})}=\frac{a_{N}}{a_{0} \mathrm{e}^{\mathrm{i} k_{z}^{(0)} L_{z}^{(0)}}}=\frac{\operatorname{det}\left(m_{N, 0}\right)}{\left(m_{N, 0}\right)_{22}} \mathrm{e}^{-\mathrm{i} k_{z}^{(0)} L_{z}^{(0)}},
$$

while for TM

$$
t_{N, 0}^{(\mathrm{TM})}=\frac{n_{0}}{n_{N}} \frac{a_{N}}{a_{0} \mathrm{e}^{\mathrm{i} k_{z}^{(0)} L_{z}^{(0)}}}=\frac{n_{0}}{n_{N}} \frac{\operatorname{det}\left(m_{N, 0}\right)}{\left(m_{N, 0}\right)_{22}} \mathrm{e}^{-\mathrm{i} k_{z}^{(0)} L_{z}^{(0)}} .
$$

The above expressions, derived as functions of elements of matrices $m_{i}$, are general and can be applied to any structure built of dielectric layers, including defected photonic crystals, chirped photonic crystals, as well as any aperiodic multilayer structure.

For a $1 \mathrm{D}$ photonic crystal, with $j$ indexing elementary cells, one defines the translation matrix $M_{j}$ :

$$
M_{j}=m_{2 j+3,2 j+2} m_{2 j+2,2 j+1} .
$$

In a particular case of the photonic crystal presented in Fig. 1, which has no defects and it is surrounded by dielectrics with refractive index $n_{1}$, all the translation matrices are equal to $M$, which is a $2 \times 2$ matrix

$$
M=\left(\begin{array}{ll}
A & B \\
C & D
\end{array}\right)
$$

An important feature of matrix $M$ is that it is unimodular, therefore one can use the Chebyshev identity

$$
M^{N}=\frac{\sin (N K \Lambda)}{\sin (K \Lambda)}\left(\begin{array}{cc}
A-\frac{\sin ((N-1) K \Lambda)}{\sin (N K \Lambda)} & B \\
C & D-\frac{\sin ((N-1) K \Lambda)}{\sin (N K \Lambda)}
\end{array}\right),
$$

where 


$$
K \Lambda=\arccos \left(\frac{A+D}{2}\right) .
$$

For TE polarization, the elements of the matrix are equal to

$$
\begin{aligned}
& A^{(\mathrm{TE})}=\mathrm{e}^{\mathrm{i} \beta_{1} a}\left[\cos \left(\beta_{2} b\right)+\frac{\mathrm{i}}{2}\left(\frac{\beta_{1}}{\beta_{2}}+\frac{\beta_{2}}{\beta_{1}}\right) \sin \left(\beta_{2} b\right)\right], \\
& B^{(\mathrm{TE})}=\frac{\mathrm{i}}{2} \mathrm{e}^{-\mathrm{i} \beta_{1} a}\left(\frac{\beta_{2}}{\beta_{1}}-\frac{\beta_{1}}{\beta_{2}}\right) \sin \left(\beta_{2} b\right), \\
& C^{(\mathrm{TE})}=\frac{\mathrm{i}}{2} \mathrm{e}^{\mathrm{i} \beta_{1} a}\left(\frac{\beta_{1}}{\beta_{2}}-\frac{\beta_{2}}{\beta_{1}}\right) \sin \left(\beta_{2} b\right), \\
& D^{(\mathrm{TE})}=\mathrm{e}^{-\mathrm{i} \beta_{1} a}\left[\cos \left(\beta_{2} b\right)-\frac{\mathrm{i}}{2}\left(\frac{\beta_{1}}{\beta_{2}}+\frac{\beta_{2}}{\beta_{1}}\right) \sin \left(\beta_{2} b\right)\right],
\end{aligned}
$$

and for TM

$$
\begin{aligned}
& A^{(\mathrm{TM})}=\mathrm{e}^{\mathrm{i} \beta_{1} a}\left[\cos \left(\beta_{2} b\right)+\frac{\mathrm{i}}{2}\left(\frac{n_{2}^{2}}{n_{1}^{2}} \frac{\beta_{1}}{\beta_{2}}+\frac{n_{1}^{2}}{n_{2}^{2}} \frac{\beta_{2}}{\beta_{1}}\right) \sin \left(\beta_{2} b\right)\right], \\
& B^{(\mathrm{TM})}=\frac{\mathrm{i}}{2} \mathrm{e}^{-\mathrm{i} \beta_{1} a}\left(\frac{n_{1}^{2}}{n_{2}^{2}} \frac{\beta_{2}}{\beta_{1}}-\frac{n_{2}^{2}}{n_{1}^{2}} \frac{\beta_{1}}{\beta_{2}}\right) \sin \left(\beta_{2} b\right), \\
& C^{(\mathrm{TM})}=\frac{\mathrm{i}}{2} \mathrm{e}^{\mathrm{i} \beta_{1} a}\left(\frac{n_{2}^{2}}{n_{1}^{2}} \frac{\beta_{1}}{\beta_{2}}-\frac{n_{1}^{2}}{n_{2}^{2}} \frac{\beta_{2}}{\beta_{1}}\right) \sin \left(\beta_{2} b\right), \\
& D^{(\mathrm{TM})}=\mathrm{e}^{-\mathrm{i} \beta_{1} a}\left[\cos \left(\beta_{2} b\right)-\frac{\mathrm{i}}{2}\left(\frac{n_{2}^{2}}{n_{1}^{2}} \frac{\beta_{1}}{\beta_{2}}+\frac{n_{1}^{2}}{n_{2}^{2}} \frac{\beta_{2}}{\beta_{1}}\right) \sin \left(\beta_{2} b\right)\right],
\end{aligned}
$$

where $\beta_{j}=k_{z}^{(j)}, n_{j}=n_{(j)}, a=L_{z}^{(1)}$, and $b=L_{z}^{(2)}$. The reflection coefficient (7) becomes then

$$
r_{2 N, 0}=\frac{B}{D-\frac{\sin ((N-1) K \Lambda)}{\sin (N K \Lambda)}}
$$

and the transmission coefficients $(8 \mathrm{a})$ and $(8 \mathrm{~b})$ become

$$
\begin{aligned}
t_{2 N, 0}^{(\mathrm{TE})} & =\frac{\left[\frac{\sin ((N-1) K \Lambda)}{\sin (N K \Lambda)}\right]^{2}-(A+D) \frac{\sin ((N-1) K \Lambda)}{\sin (N K \Lambda)}+A D-B C}{\frac{\sin (K \Lambda)}{\sin (N K \Lambda)}\left[D-\frac{\sin ((N-1) K \Lambda)}{\sin (N K \Lambda)}\right]}, \\
t_{2 N, 0}^{(\mathrm{TM})} & =\frac{n_{0}}{n_{2 N}} \frac{\left[\frac{\sin ((N-1) K \Lambda)}{\sin (N K \Lambda)}\right]^{2}-(A+D) \frac{\sin ((N-1) K \Lambda)}{\sin (N K \Lambda)}+A D-B C}{\frac{\sin (K \Lambda)}{\sin (N K \Lambda)}\left[D-\frac{\sin ((N-1) K \Lambda)}{\sin (N K \Lambda)}\right]} .
\end{aligned}
$$

It is easy to see that the change of sign of $k_{z}^{(0)}$ causes the $m_{i+1, i}$ matrix to become its complex conjugate 


$$
k_{z}^{(0)} \rightarrow-k_{z}^{(0)} \Rightarrow m_{i+1, i} \rightarrow m_{i+1, i}^{*}
$$

The same applies then to the reflection and transmission coefficients

$$
r_{N, 0}\left(\boldsymbol{k}_{*}\right)=r_{N, 0}(-\boldsymbol{k})=r_{N, 0}^{*}(\boldsymbol{k})
$$

and

$$
t_{N, 0}\left(\boldsymbol{k}_{*}\right)=t_{N, 0}(-\boldsymbol{k})=t_{N, 0}^{*}(\boldsymbol{k}) .
$$

\section{Effective resonator model}

Having found the reflection coefficients (11) (or more general (7)) for both boundary planes of a layer of a photonic crystal, it is possible to replace the layer with an equivalent resonator filled with the layer's dielectric, with width $L_{z}$ the same as the layer's one and with two mirrors, whose reflection coefficients are the same as of the layer's boundaries. This idea is illustrated in Fig. 2. The problem of finding electric field in the photonic crystal is then replaced with an equivalent problem of finding electric field in the resonator.

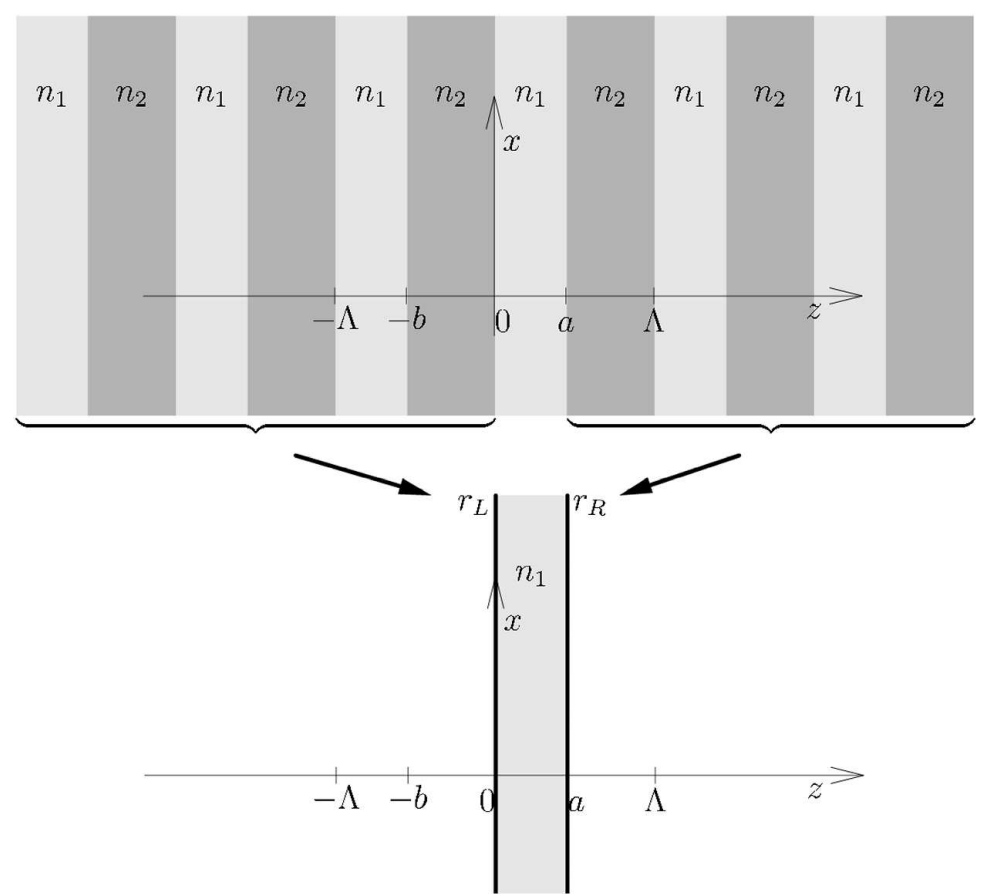

Fig. 2. Illustration of an effective resonator model.

Let $r_{\mathrm{L}}$ be the reflection coefficient of the "left" mirror, and $r_{\mathrm{R}}$ - of the "right" one. If the resonator is excited with a plane wave $\boldsymbol{E}_{0}(\boldsymbol{r})$ with wave vector $\boldsymbol{k}$ and polarization $\epsilon$ either TE or TM: 


$$
\boldsymbol{E}_{0}(\boldsymbol{r})=A_{0} \boldsymbol{e}_{k \epsilon} \mathrm{e}^{\mathrm{i} \boldsymbol{k} \boldsymbol{r}},
$$

then this wave is incident on the "right" mirror, which causes a reflected wave to appear

$$
\boldsymbol{E}_{0}^{\prime}(\boldsymbol{r})=r_{\mathrm{R}} A_{0} \boldsymbol{e}_{k_{*} \epsilon} \mathrm{e}^{\mathrm{i} \boldsymbol{k}_{*} \boldsymbol{r}} \mathrm{e}^{2 \mathrm{i} k_{z} L_{z}} .
$$

The reflection of this wave from the "left" mirror causes another plane wave to emerge

$$
\boldsymbol{E}_{1}(\boldsymbol{r})=r_{\mathrm{L}} r_{\mathrm{R}} A_{0} \boldsymbol{e}_{k \epsilon} \mathrm{e}^{\mathrm{i} \boldsymbol{k} \boldsymbol{r}} \mathrm{e}^{2 \mathrm{i} k_{z} L_{z}} .
$$

As next reflections follow, a series of plane waves with wave vectors $\boldsymbol{k}$ and $\boldsymbol{k}_{*}$ appears in the resonator

$$
\boldsymbol{E}_{+}(\boldsymbol{r})=\boldsymbol{E}_{0}^{\prime}(\boldsymbol{r})+\left[\boldsymbol{E}_{0}(\boldsymbol{r})+\boldsymbol{E}_{0}^{\prime}(\boldsymbol{r})\right] \sum_{j=1}^{\infty}\left(r_{\mathrm{L}} r_{\mathrm{R}} \mathrm{e}^{2 \mathrm{i} k_{z} L_{z}}\right)^{j} .
$$

However, there appears a second series of plane waves, because the first plane wave, $\boldsymbol{E}_{0}$, has to satisfy boundary conditions on the "left" mirror as well, which means that there appears a plane wave

$$
\boldsymbol{E}_{-1}^{\prime}(\boldsymbol{r})=r_{\mathrm{L}}^{*} A_{0} \boldsymbol{e}_{k_{*} \epsilon} \mathrm{e}^{\mathrm{i} \boldsymbol{k}_{*} \boldsymbol{r}},
$$

which gives rise to a plane wave

$$
\boldsymbol{E}_{-1}(\boldsymbol{r})=r_{\mathrm{L}}^{*} r_{\mathrm{R}}^{*} A_{0} \boldsymbol{e}_{k_{*} \epsilon} \mathrm{e}^{\mathrm{i} \boldsymbol{k}_{*} \boldsymbol{r}} \mathrm{e}^{-2 \mathrm{i} k_{z} L_{z}} .
$$

The resulting second series of plane waves is

$$
\boldsymbol{E}_{-}(\boldsymbol{r})=\left[\boldsymbol{E}_{-1}(\boldsymbol{r})+\boldsymbol{E}_{-1}^{\prime}(\boldsymbol{r})\right] \sum_{j=0}^{\infty}\left(r_{\mathrm{L}}^{*} r_{\mathrm{R}}^{*} \mathrm{e}^{-2 \mathrm{i} k_{z} L_{z}}\right)^{j} .
$$

The effective electric field in the resonator is the sum of these series and the origin plane wave

$$
\boldsymbol{E}(\boldsymbol{r})=\boldsymbol{E}_{0}(\boldsymbol{r})+\boldsymbol{E}_{+}(\boldsymbol{r})+\boldsymbol{E}_{-}(\boldsymbol{r}) .
$$

A simple calculation shows that the electric field in the resonator, which includes all spectral components with both polarizations, can be written as

$$
\boldsymbol{E}(\boldsymbol{r}, t)=\sum_{\epsilon} \int \mathrm{d}^{3} k \rho_{\epsilon}(\boldsymbol{k}) A_{k \epsilon}(t)\left[\boldsymbol{e}_{k \epsilon} \mathrm{e}^{\mathrm{i} \boldsymbol{k} \boldsymbol{r}}+\boldsymbol{e}_{k_{*} \epsilon} \mathrm{e}^{\mathrm{i} \boldsymbol{k}_{*} \boldsymbol{r}} \xi_{\epsilon}^{*}(\boldsymbol{k})\right],
$$

in which expression $\rho_{\epsilon}(\boldsymbol{k})$ is the mode spectrum of the layer

$$
\rho_{\epsilon}(\boldsymbol{k})=\frac{1}{8 \pi^{3}} \frac{1-\left|r_{\mathrm{L} \epsilon}(\boldsymbol{k}) r_{\mathrm{R} \epsilon}(\boldsymbol{k})\right|^{2}}{1+\left|r_{\mathrm{L} \epsilon}(\boldsymbol{k}) r_{\mathrm{R} \epsilon}(\boldsymbol{k})\right|^{2}-\operatorname{Re}\left[r_{\mathrm{L} \epsilon}(\boldsymbol{k}) r_{\mathrm{R} \epsilon}(\boldsymbol{k}) \mathrm{e}^{2 \mathrm{i} k_{z} L_{z}}\right]}
$$

(normalization is explained in [10]) and $\xi_{\epsilon}(\boldsymbol{k})$ is the coupling function

$$
\xi_{\epsilon}(\boldsymbol{k})=\frac{r_{\mathrm{R} \epsilon}^{*}(\boldsymbol{k})\left[1-\left|r_{\mathrm{L} \epsilon}(\boldsymbol{k})\right|^{2}\right] \mathrm{e}^{-2 \mathrm{i} k_{z} L_{z}}+r_{\mathrm{L} \epsilon}(\boldsymbol{k})\left[1-\left|r_{\mathrm{R} \epsilon}(\boldsymbol{k})\right|^{2}\right]}{1-\left|r_{\mathrm{L} \epsilon}(\boldsymbol{k}) r_{\mathrm{R} \epsilon}(\boldsymbol{k})\right|^{2}} .
$$

Magnetic field vector can be expressed with the formula

$$
\boldsymbol{H}(\boldsymbol{r}, t)=\sum_{\epsilon} \int \mathrm{d}^{3} k \rho_{\epsilon}(\boldsymbol{k}) B_{k \epsilon}(t)\left[\boldsymbol{k} \times \boldsymbol{e}_{k \epsilon} \mathrm{e}^{\mathrm{i} \boldsymbol{k} \boldsymbol{r}}+\boldsymbol{k}_{*} \times \boldsymbol{e}_{k_{*} \epsilon} \mathrm{e}^{\mathrm{i} \boldsymbol{k}_{*} \boldsymbol{r}} \xi_{\epsilon}^{*}(\boldsymbol{k})\right] .
$$




\section{Electromagnetic field in a $1 \mathrm{D}$ photonic crystal}

If the electric field in the $i$-th layer of the photonic crystal is given by the formula (23), then electric field in $j$-th layer $\boldsymbol{E}_{j}(\boldsymbol{r}, t)$ can be expressed as the following:

$$
\begin{aligned}
& \boldsymbol{E}_{j}(\boldsymbol{r}, t)= \\
& \quad \sum_{\epsilon} \int \mathrm{d}^{3} k \rho_{\epsilon}(\boldsymbol{k}) A_{k \epsilon}(t)\left[u_{j k \epsilon} \boldsymbol{e}_{k^{(j)}} \epsilon \mathrm{e}^{\mathrm{i} \boldsymbol{k}^{(j)} \boldsymbol{r}^{(j)}}+v_{j k_{*} \epsilon} \boldsymbol{e}_{k_{*}^{(j)} \epsilon} \mathrm{e}^{\mathrm{i} \boldsymbol{k}_{*}^{(j)} \boldsymbol{r}^{(j)}}\right],
\end{aligned}
$$

where $\boldsymbol{r}^{(j)}$ denotes position in the $j$-th layer's coordinate system. For $j>i$ :

$$
\begin{aligned}
& u_{j k \epsilon}=\left(\delta_{\epsilon \mathrm{TE}}+\frac{n_{i}}{n_{j}} \delta_{\epsilon \mathrm{TM}}\right)\left[\left(m_{j, i}^{\epsilon}(\boldsymbol{k})\right)_{11}+\left(m_{j, i}^{\epsilon}(\boldsymbol{k})\right)_{12} \xi_{\epsilon}^{*}(\boldsymbol{k})\right], \\
& v_{j k \epsilon}=\left(\delta_{\epsilon \mathrm{TE}}+\frac{n_{i}}{n_{j}} \delta_{\epsilon \mathrm{TM}}\right)\left[\left(m_{j, i}^{\epsilon}\left(\boldsymbol{k}_{*}\right)\right)_{21}+\left(m_{j, i}^{\epsilon}\left(\boldsymbol{k}_{*}\right)\right)_{22} \xi_{\epsilon}(\boldsymbol{k})\right],
\end{aligned}
$$

with matrix $m_{j, i}^{\epsilon}(\boldsymbol{k})$ defined by $(6 \mathrm{~b})$ with explicitly indicated dependence on polarization and wave vector, and for $j<i$ :

$$
\begin{aligned}
& u_{j k \epsilon}=\left(\delta_{\epsilon \mathrm{TE}}+\frac{n_{i}}{n_{j}} \delta_{\epsilon \mathrm{TM}}\right)\left[\left(w_{j, i}^{\epsilon}(\boldsymbol{k})\right)_{11}+\left(w_{j, i}^{\epsilon}(\boldsymbol{k})\right)_{12} \xi_{\epsilon}^{*}(\boldsymbol{k})\right], \\
& v_{j k \epsilon}=\left(\delta_{\epsilon \mathrm{TE}}+\frac{n_{i}}{n_{j}} \delta_{\epsilon \mathrm{TM}}\right)\left[\left(w_{j, i}^{\epsilon}\left(\boldsymbol{k}_{*}\right)\right)_{21}+\left(w_{j, i}^{\epsilon}\left(\boldsymbol{k}_{*}\right)\right)_{22} \xi_{\epsilon}(\boldsymbol{k})\right],
\end{aligned}
$$

with

$$
w_{j, i}^{\epsilon}(\boldsymbol{k})=w_{j, j+1}^{\epsilon}(\boldsymbol{k}) \ldots w_{i-1, i}^{\epsilon}(\boldsymbol{k}),
$$

where $w_{i-1, i}^{\epsilon}(\boldsymbol{k})$ is defined by $(5 \mathrm{~b})$. Magnetic field associated with electric field (27) can be found directly from the Maxwell equations.

Envelopes of electric field in a photonic crystal for different modes are presented in Figs. 3-6. All the calculations were made for a photonic crystal with $n_{1}=1.5, n_{2}=1, a=b=0.25 \mu \mathrm{m}$, consisting of 80 elementary cells. A plane wave was excited in a layer with refractive index $n_{1}$ extending from $z=0 \mu \mathrm{m}$ to $z=0.25 \mu \mathrm{m}$. The results were obtained for perpendicular incidence onto the layer's boundaries (wave vector $\boldsymbol{k} \| \boldsymbol{e}_{z}$ ). Calculations have been also made for a defected photonic crystal, in which case the defect was introduced by a change of width of one of the crystal's $n_{1}$ layers by $\Delta a=0.1 \mu \mathrm{m}$.

Figure 3 depicts envelope of a mode propagating in the photonic crystal (a plane wave with frequency $f=300 \mathrm{THz}$ ). The envelope repeats its values quite regularly in consecutive elementary cells. In this case there is no region of the crystal that would be privileged in any way. For a photonic crystal with a defect (Fig. 4), the envelope behaves similarly as for the crystal with no defects, with one important difference — the defect affects the propagation reflecting a part of the propagating wave. Therefore, the envelope has different maximal values in the regions before and behind the defect. 


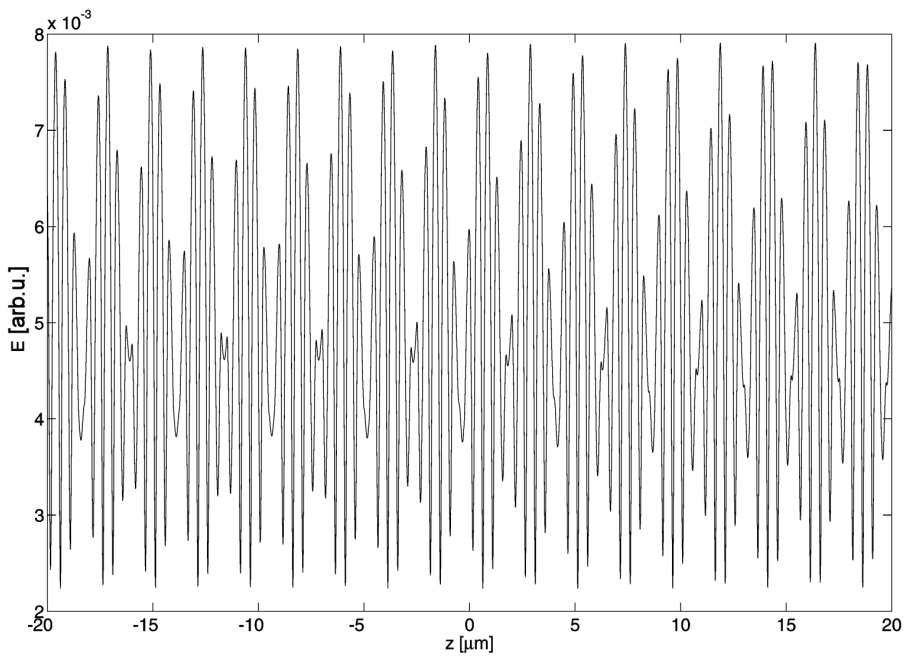

Fig. 3. Envelope of electric field for a propagating mode.

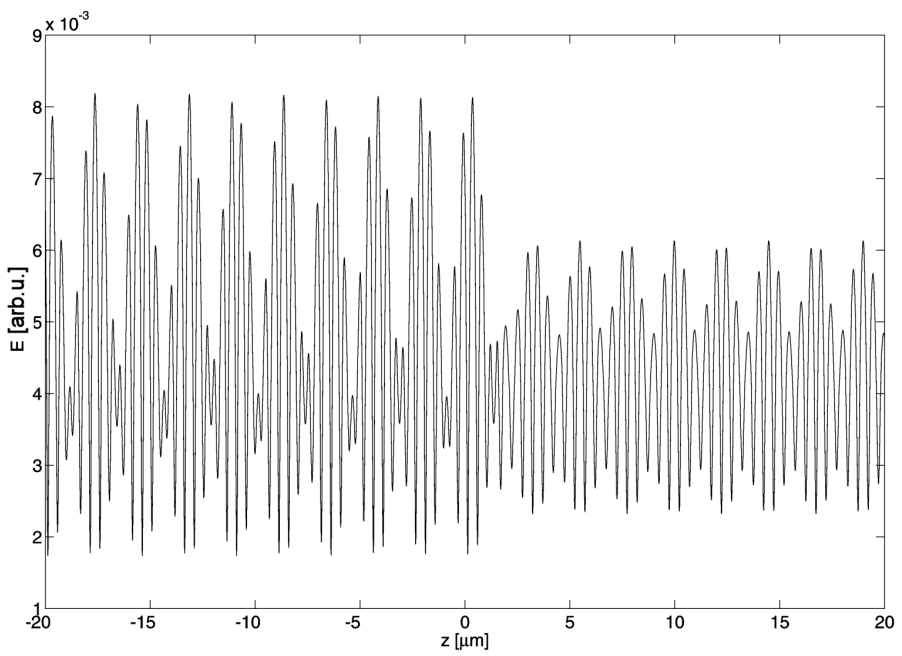

Fig. 4. Envelope of electric field for a propagating mode in a photonic crystal with a defect.

The envelope associated with a plane wave with frequency from the photonic band gap $(f=483.473 \mathrm{THz})$ is presented in Fig. 5 . Such a wave cannot exist in an infinite photonic crystal, however, a finite crystal does not reflect $100 \%$ of the wave and therefore cannot fully extinguish its electromagnetic field. The envelope is very small and it rises near the edges of the photonic crystal. This happens because a wave in the middle of the crystal is reflected from a greater number of layers and these reflections are effectively stronger, thus allowing a more destructive interference. A defect can, however, introduce a phase shift, 


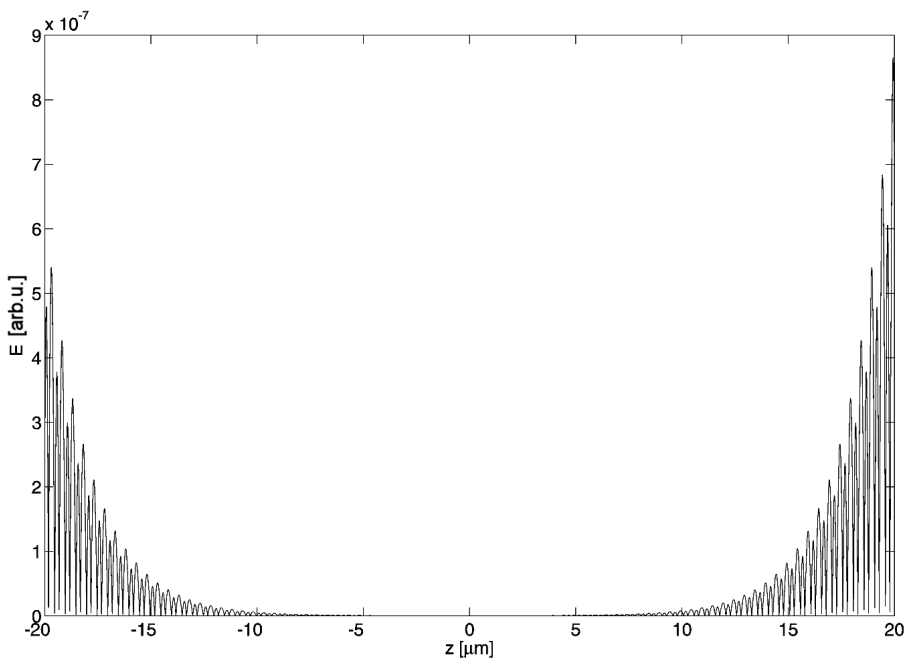

Fig. 5. Envelope of electric field for a wave from a photonic band gap.

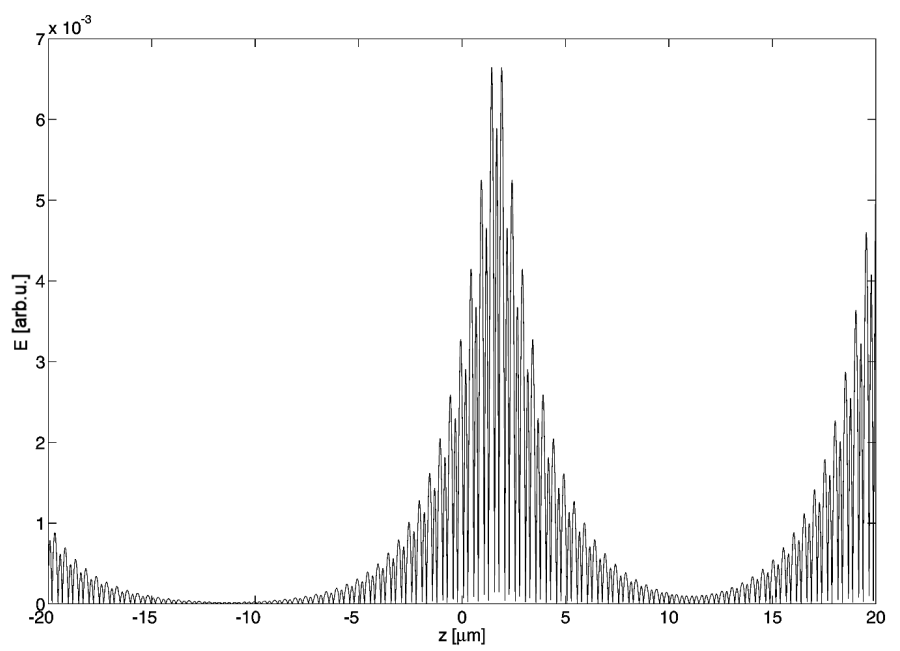

Fig. 6. Envelope of electric field for a defect mode.

which causes the interference to become constructive in a certain region near the defect. Electromagnetic field in the photonic crystal becomes then stronger, and is particularly strong in that region - there appears a defect mode, associated with the existence of the defect. This behaviour of electromagnetic field is illustrated in Fig. 6. 


\section{References}

[1] E. Yablonovitch, Phys. Rev. Lett. 58, 2059 (1987).

[2] S. John, Phys. Rev. Lett. 58, 2486 (1987).

[3] S. Fan, J.N. Winn, A. Devenyi, J.C. Chen, R.D. Meade, J.D. Joannopoulos, J. Opt. Soc. Am. B 12, 1267 (1995).

[4] A. Mekis, J.C. Chen, I. Kurland, S. Fan, P.R. Villeneuve, J.D. Joannopoulos, Phys. Rev. Lett. 77, 3787 (1996).

[5] J.C. Chen, H.A. Haus, S. Fan, P.R. Villeneuve, J.D. Joannopoulos, J. Lightwave Technol. 14, 2575 (1996).

[6] I.S. Fogel, J.M. Bendickson, M.D. Tocci, M.J. Bloemer, M. Scalora, C.M. Bowden, J.P. Dowling, Pure Appl. Opt. 7, 393 (1998).

[7] M. Boroditsky, R. Vrijen, T.F. Krauss, R. Coccioli, R. Bhat, E. Yablonovitch, J. Lightwave Technol. 17, 2096 (1999).

[8] P. Szczepański, IEEE J. Quantum Electron. 24, 1248 (1988).

[9] P. Yeh, A. Yariv, C. Hong, J. Opt. Soc. Am. 67, 423 (1977).

[10] A. Rudziński, A. Tyszka-Zawadzka, P. Szczepański, Proc. SPIE 5950, 59501A (2005). 